éphéméride, on fournira le résidu correspondant à chaque minimum observé, ainsi que la formule numérique ayant servi au calcul de l'éphéméride.

$2^{\circ}$. Pour rémédier autant que possible au manque d'observations pendant les dernières années, il est recommandé de déterminer, au cours des deux ou trois années à venir, les minima de toutes les variables à éclipses intéressantes, non seulement de celles qui offrent un mouvement de la ligne des apsides, mais encore de celles dont la période semble présenter des fluctuations. Les observations visuelles peuvent rendre ici de grands services.

$3^{\circ}$. L'étude détaillée des systèmes binaires à éclipses les plus intéressants exigeant la connaissance de leurs variations spectrales, il est recommandé de mener autant que possible simultanément les observations photométriques et les observations spectrographiques. Les unes et les autres devront être concentrées sur le plus court intervalle de temps possible. La même recommandation s'applique aussi à l'étude des céphéides brillantes, pour lesquelles on peut obtenir des spectrogrammes à grande dispersion.

5. La Commission signale l'importance des observations spectrographiques d'étoiles variables dans l'hémisphère sud.

6. La Commission a approuvé les règles pour la dénomination des étoiles variables, qui ont été provisoirement adoptées par la sous-commission de la dénomination des étoiles variables.

Ces règles sont:

Doivent être dénommées:

(a) les variables périodiques dont le type, la période et la courbe de lumière sont connus;

(b) les variables non périodiques dont le type a été bien établi;

(c) les variables dont le type paraît être évident, bien qu'aucun autre détail ne soit donné.

Le choix des étoiles, satisfaisant aux règles mentionnées ci-dessus, doit reposer sur une discussion stricte de toutes les données.

7. La Commission propose que la subvention annuelle accordée à M. Banachiewicz pour la publication des éphémérides des étoiles doubles à éclipses, soit continuée à raison de rooo (mille) francs-or, à la condition que les dates soient données exclusivement dans la période julienne.

8. La Commission a décidé de demander à l'Assemblée Générale de faire imprimer et distribuer une traduction en anglais de l'introduction, de la liste des auteurs russes et des remarques du nouveau catalogue général des étoiles variables, publié par MM. Kukarkin et Parenago à la demande de 1'U.A.I. Le nombre des pages données en langue et caractères russes est d'environ vingt. Une subvention unique de 250 dollars serait nécessaire probablement.

9. La Commission a décidé de demander à l'Assemblée Générale de faire imprimer et distribuer la 44ième liste des noms d'étoiles variables, dont le manuscrit a été préparé par MM. Kukarkin et Parenago. La liste contient la dénomination de 266 étoiles variables nouvelles. Une subvention annuelle de 250 dollars suffira probablement pour couvrir les frais.

I0. La Commission demande que le Bureau de l'Union entreprenne la publication de la liste des observations inédites des étoiles variables, dont M. Grouiller avait été chargé en r938. M. Brun a communiqué que le manuscrit se trouve à l'Observatoire de Lyon prêt pour la publication.

II. La Commission approuve la formation d'une commission nouvelle (42) sur les étoiles doubles photométriques.

\title{
Commission 28. (NÉBULEUSES EXTRA-GALACTIQUES)
}

La Commission attire l'attention sur le fait que les remarquables photographies de nébuleuses faites par le docteur C. O. Lampland de l'Observatoire Lowell n'ont pas encore 
été publiées. La Commission considère qu'il serait d'une grande valeur scientifique de publier au moins des photographies sélectionnées parmi ce matériel étendu. Elle espère sincèrement que les fonds nécessaires à cette publication pourront être obtenus de l'une ou l'autre des organisations astronomiques américaines intéressées.

\section{Commission 29. (Spectres STellaires)}

I. La Commission recommande au Comité Exécutif que la Sous-commission des spectres des novae soit étendue afin d'embrasser les spectres de toutes les étoiles variables; que la Commission 27 (Etoiles variables) ainsi que la Commission 29 (Spectres stellaires) désignent chacune deux ou trois membres qui feraient partie de cette nouvelle souscommission, et que son nom soit: 'Sous-commission des spectres des étoiles variables.'

2. La Commission recommande qu'une compilation et un atlas des spectres moléculaires obtenus au laboratoire présentant un intérêt astrophysique soient faits et publiés aussitôt que possible.

3. La Commission recommande que le projet de M. Schwassmann de photographier le ciel entier avec de grands télescopes Schmidt pourvus d'un prisme objectif soit exécuté autant que possible. Si plusieurs observatoires collaborent à ce plan, des rapports ainsi qu'un échange d'informations seront nécessaires. Le président de la Commission 29 servira d'intermédiaire.

\section{Commission 3I. (HEURE)}

I. M. A. Danjon est proposé comme représentant de la Commission 3I (C.I.H.) auprès du C.C.I.R. (Comité Consultatif International des Radiocommunications).

2. La Commission recommande que les observateurs veuillent bien mentionner en publiant leurs résultats, si les observations ont été faites dans les deux positions de la lunette et s'il a été tenu compte de l'inégalité et des irrégularités des tourillons.

3. La Commission recommande, suivant la suggestion qui lui est faite par l'U.R.S.I. (Union Radio-Scientifique Internationale), que les astronomes déterminent, en collaboration avec les radio-électriciens, la vitesse de propagation des ondes longues. Dans ce but, il serait très désirable que la station d'émission d'Annapolis (N.S.S.) reprenne ses émissions horaires sur ondes longues ( $16.000 \mathrm{~m}$.).

4. La Commission émet le vœu que le Bulletin Horaire reprenne la publication des comparaisons mutuelles des garde-temps, publication qui a été interrompue en I936.

5. Il serait souhaitable que les observatoires qui commandent les émissions des signaux horaires scientifiques prennent toutes dispositions pour que, d'un jour à l'autre, les corrections de ces signaux ne varient pas d'une manière trop sensible. (Cette demande est faite par les géodésiens qui désirent déterminer immédiatement les marches de leurs garde-temps.)

6. Une subvention annuelle de $\$ 4.400$ est demandée pour le fonctionnement du Bureau International de l'Heure.

\section{Commission 34. (MatiÈre interstellaire et Nébuleuses Galactigues)*}

Les spectres des étoiles des premiers types dans l'hémisphère sud devront être déterminés aussitôt que possible.

* Cette résolution a été reçue par le Secrétaire Général après la dernière Assemblée Générale; par conséquent elle n'a pas pu être approuvée par celle-ci. 